\title{
Sarcoïdose jugale : à propos d'un cas
}

\section{Sarcoidosis of the cheek: case report}

\author{
PIERRE JACQUEMART ${ }^{*}$, BENJAMIN BAJER ${ }^{*}$, JOELLE LAEDRICH ${ }^{\star *}$, MARC DUFET $^{*}$
}

\section{RÉSUMÉ}

La sarcoïdose est une granulomatose multisystémique, de localisation buccale rare. On rapporte un cas où la découverte d'une lésion labio-jugale chez un homme de 44 ans a ensuite permis la prise en charge globale l'affection. En l'absence de spécificité clinique de la lésion buccale, le diagnostic a été évoqué sur l'examen anatomopathologique. Les examens complémentaires ont confirmé le diagnostic et révélé d'autres localisations. Le traitement général, efficace, a donné lieu à des effets secondaires, en particulier un diabète corticoinduit. (Med Buccale Chir Buccale 2004; 10: 83-89)

mots clés : Sarcoïdose, granulomatose, complication.

médecine buccale chirurgie buccale

VOL. $10, \mathrm{~N}^{\circ} 2$ 2004

page 83

\section{SUMMARY}

Sarcoidosis is a multisytemic granulatosis disease with uncommon oral location. A swelling located in the cheek of a 44 years old man is reported here. The symptomatology was not specific but the biopsy found a muscular location in the cheek. Complémentary findings made the evidence of the illness. The swelling relieved in one month with general coticotheray. But side effects was decompensation of a diabetes and possibly an enotional collapse. (Med Buccale Chir Buccale 2004; 10: 83-89)

key words : Sarcoidosis, granulomatosis lesion, complication.

\footnotetext{
* Service de stomatologie, Hôpital André Grégoire, 93105 Montreuil, France

* * Service de néphrologie et d'hémodialyse, Hôpital André Grégoire, 93105 Montreuil, France

Demande de tirés à part :

Dr Pierre Jacquemart Service de Stomatologie CHI André Grégoire 56 bd de la Boissière 93105 Montreuil
} 
médecine

buccale

chirurgie

buccale

VOL. $10, \mathrm{~N}^{\circ} 2$ 2004

page 84
La sarcoïdose est une affection peu fréquente, le plus souvent bénigne, présentant rarement une localisation bucco-faciale. Le tableau clinique comporte classiquement des lésions pulmonaires et des adénopathies médiastinales. Le diagnostic s'appuie habituellement sur un nombre limité d'éléments, le plus déterminant étant l'examen anatomopathologique. Le cas clinique présenté comporte une lésion buccale de sarcoïdose qui conduit à la découverte d'autres lésions systémiques. Dans cette localisation initiale, le rôle de l'odonto-stomatologiste reste important pour le dépistage, même si le caractère systémique de la maladie implique une prise en charge médicale. Ce cas illustre aussi les difficultés thérapeutiques rencontrées, en particulier dans l'évaluation du rapport bénéfice-risque.

\section{CAS CLINIQUE}

Un homme de 44 ans vient consulter pour une cellulite génienne gauche chronique dont le début semble remonter à 4 ans. Les antécédents révèlent une crise comitiale isolée dans l'adolescence, une toxicomanie ancienne par voie nasale et une intoxication tabagique chronique à 10 paquets/année.

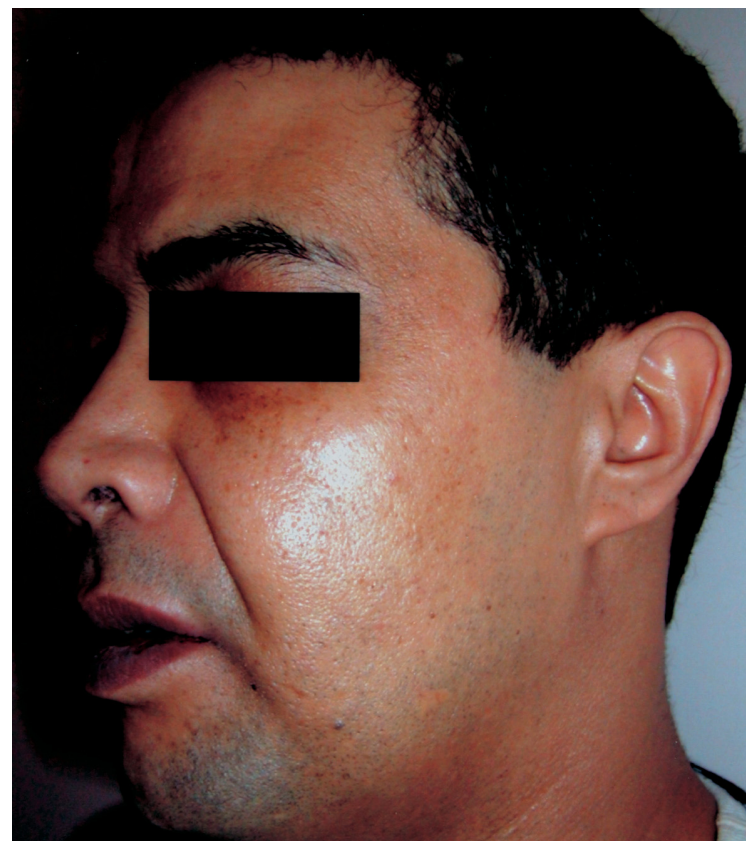

Figure 1 : Vue de profil : le revêtement cutané est d'aspect normal.

Lateral view of the face showing the usual aspect of the skin.
Dans les antécédents familiaux, on note qu'une sœur est diabétique insulino-dépendante et que la mère est atteinte de la maladie d'Alzheimer. L'examen clinique met en évidence une tuméfaction indolore provoquant une asymétrie faciale et une asymétrie labiale visible lors de l'ouverture buccale, associée à un léger trismus (Fig. 1-2). Le vestibule supérieur gauche est libre et l'aspect du revêtement cutané normal. A la palpation cervicale, il n'y a pas d'adénopathies mais la palpation bidigitale de la joue gauche retrouve un épaississement associé à une induration. Le patient est apyrétique. La radiographie panoramique met en évidence une image radio-claire, bien limitée, dans la région apicale de 21 à 24 et de petites images radio-opaques évoquant de la pâte d'obturation canalaire (Fig. 3). Initialement, le diagnostic de cellulite chronique d'origine dentaire est évoqué. Un traitement par amoxi-

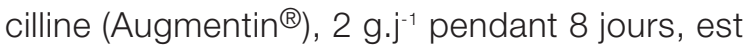
instauré et une intervention visant à cureter le foyer infectieux est programmée. Le patient est revu une semaine plus tard : la tuméfaction génienne persiste inchangée. II est alors décidé de l'hospitaliser. La numération sanguine met en évidence une leucocytose modérée. L'examen tomodensitométrique

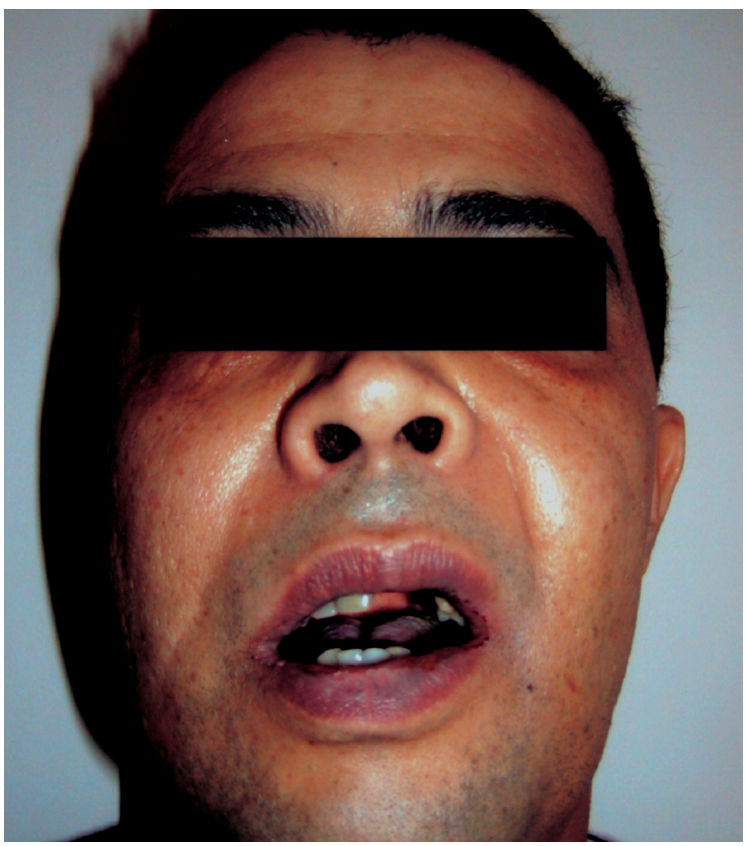

Figure 2 : Vue de face : tuméfaction génienne avec asymétrie labiale.

Front view of the face showing swelling and lip asymetry. 


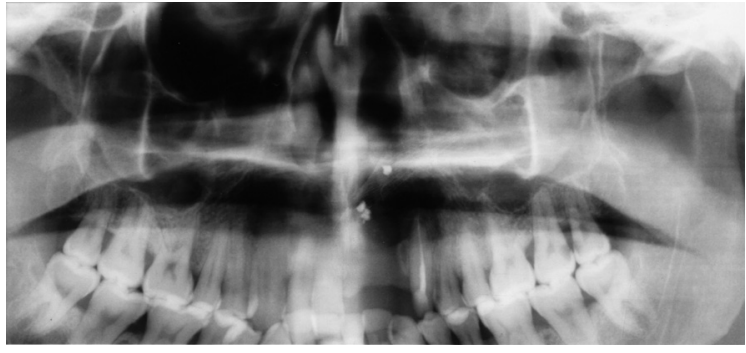

Figure 3 : Radiographie panoramique montrant une lésion ostéolytique bien limitée dans la région 21 -24. Panorex showing an osteolytic unilocular defect of the upper maxila.

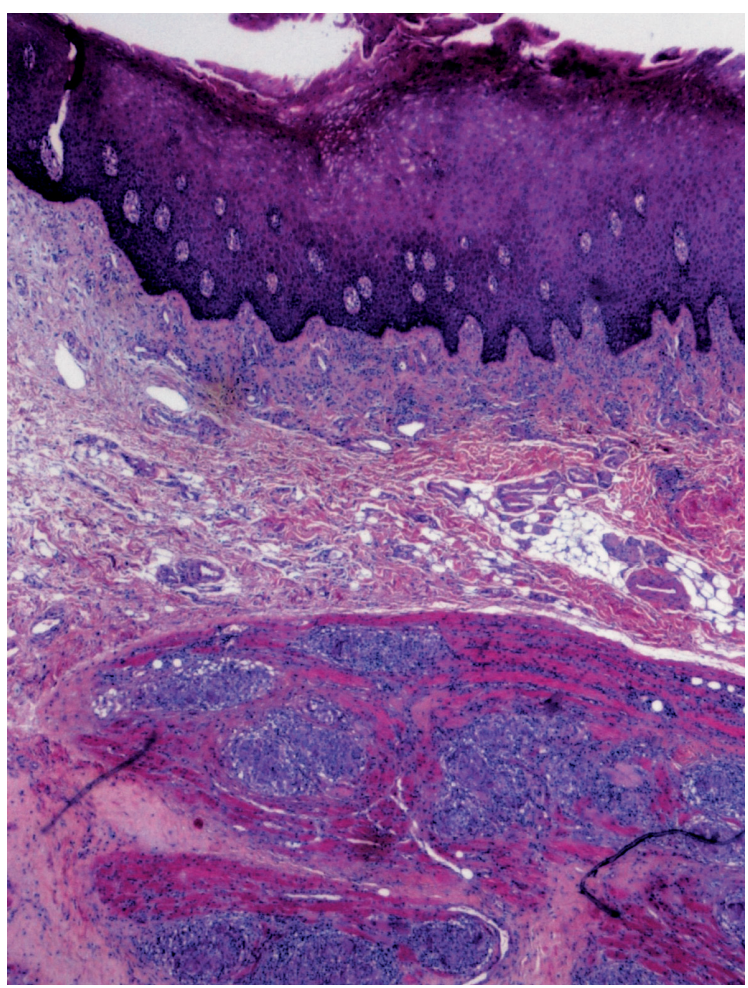

Figure 5 : Aspect histologique de la lésion jugale, avec plusieurs granulomes apparaissant dans les couches musculaires. La coloration à l'hématine colore en bleu les noyaux, l'éosine en rose le cytoplasme et les fibres musculaires, le safran en jaune le tissu conjonctif (coloration HES, X25).

Histological view showing granulatosis lesions in muscular layer (X 25).

du maxillaire (Fig. 4) confirme la présence d'un corps étranger et d'une lyse osseuse dans la région 21 à 24 et révèle l'existence d'une opacité polypoïde au pôle antéro-inférieur du sinus maxillaire gauche. Au terme du bilan, on conclut à une cellulite sur corps étranger. Il est décidé de procéder à un cure-

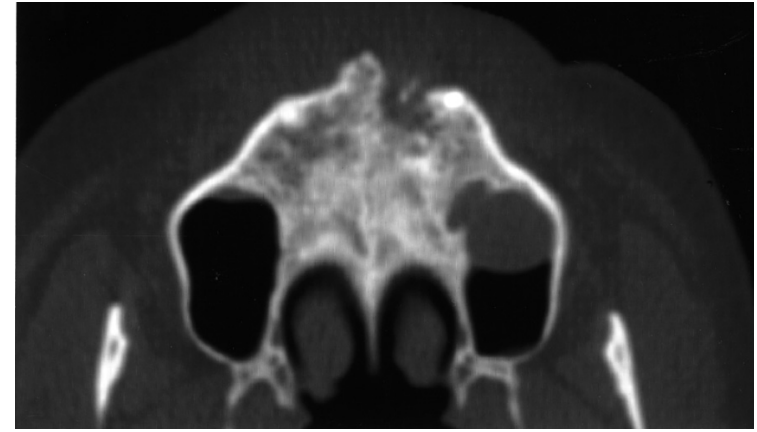

Figure 4 : Examen tomodensitométrique montrant les limites irrégulières de la lésion ostéolytique et une lésion polypoïde dans le sinus maxillaire gauche.

Axial view CT Scan showing an osteolytic unilocular defect of the upper maxilla.

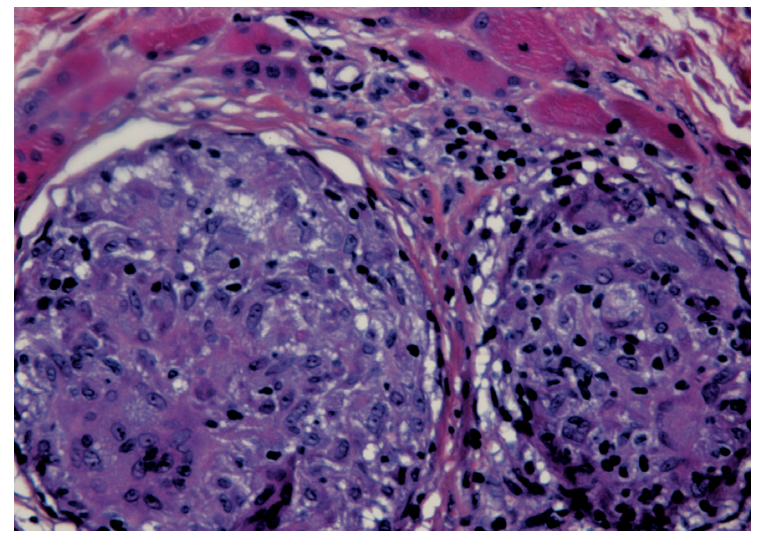

Figure 6 : Détail des granulomes pour mettre en évidence les cellules géantes (coloration HES, X 200). Histological view showing detail of granulatosis lesion with geant cells (X 200).

tage de la lésion osseuse et d'effectuer, dans le même temps, une biopsie jugale et sinusienne. L'anatomopathologiste conclut, pour le prélèvement jugal, à la présence d'une réaction granulomateuse épithélioïde et giganto-cellulaire, floride, sans nécrose caséeuse associée, envahissant les fibres musculaires, devant faire rechercher en premier lieu une sarcoïdose (Fig. 5-6) ; les fragments provenant de la biopsie sinusienne sont non spécifiques.

Des investigations complémentaires sont alors entreprises dans le cadre d'un bilan de sarcoïdose. L'enzyme de conversion et la calcémie sont normales, il n'y a pas de BK dans les crachats. Le bilan urinaire révèle la présence de protéines $\left(0.48{\left.\mathrm{~g} . \mathrm{I}^{-1}\right)}^{-1}\right.$ faisant suspecter une atteinte rénale ; la recherche médecine

buccale

chirurgie

buccale

VOL. $10, \mathrm{~N}^{\circ} 2$ 2004

page 85 
médecine

buccale

chirurgie

buccale

VOL. $10, \mathrm{~N}^{\circ} 2$ 2004

page 86 et le typage d'une immunoglobuline et de la protéine de Bence-Jones s'avèreront négatifs. L'examen tomodensitométrique thoracique (Fig. 7) révèle la présence d'adénopathies médiastinales non spécifiques compte tenu de leur taille infra-centimétrique, de bronchectasies dans le lobe moyen, d'un micronodule dans le lobe moyen et d'un autre dans le lobe inférieur. L'échographie confirme les adénopathies thoraciques. Le diagnostic de sarcoïdose systémique est alors posé et une corticothérapie (1 mg. $\left.\mathrm{kg}^{-1} \cdot \mathrm{j}^{-1}\right)$ instaurée. Après un mois de traitement, la tuméfaction jugale a complètement disparu et le bilan objective une diminution de la

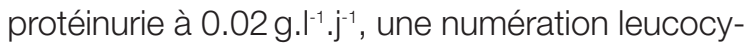

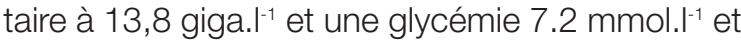

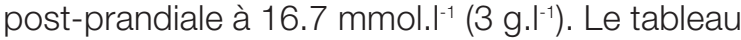
clinique comporte une asthénie, une irritabilité et un syndrome anxio-dépressif, une polydipsie, une polyurie (jusqu'à 10 mictions par nuit) et une légère dyspnée. Le diabète est pris en charge, le traitement de la sarcoïdose réévalué - le Cortancy ${ }^{\circledR}$ est ramené à $75 \mathrm{mg}^{-1} \mathrm{j}^{-1}$, puis progressivement à $50 \mathrm{mg}^{-\mathrm{j}^{-1}}$ - et, pour la dépression, on prescrit $1 \mathrm{cp}$ de Deroxat ${ }^{\circledR}$ le matin pendant quelques semaines. L'état général et l'asthénie s'améliorent, le bilan pulmonaire redevient normal mais la glycémie reste toujours élevée. La corticothérapie, réduite à $20 \mathrm{mg} . \mathrm{j}^{-1}$, sera poursuivie pendant 18 mois.

\section{DISCUSSION}

La sarcoïdose, ou maladie de Besnier-BoeckShaumann, est une affection granulomateuse multisystémique, rare, 6-7 cas par 100000 habitants en France. D'étiologie inconnue, elle serait liée à une altération de l'hypersensibilité retardée à médiation cellulaire. Anatomopathologiquement, elle se caractérise par des granulomes épithélioïdes multiples, entourant des cellules géantes multinucléées, sans nécrose caséeuse, comportant aussi des macrophages et, à leur périphérie, des lymphocytes T. Cliniquement, cette affection présente un grand polymorphisme dépendant des localisations viscérales et du degré de diffusion des lésions. La sarcoïdose comporte peu de signes généraux. Dans près de $90 \%$ des cas, il existe une atteinte pulmonaire parenchymateuse intersticielle associée, dans $33 \%$ des cas, à des signes fonctionnels respiratoires. Les adénopa-

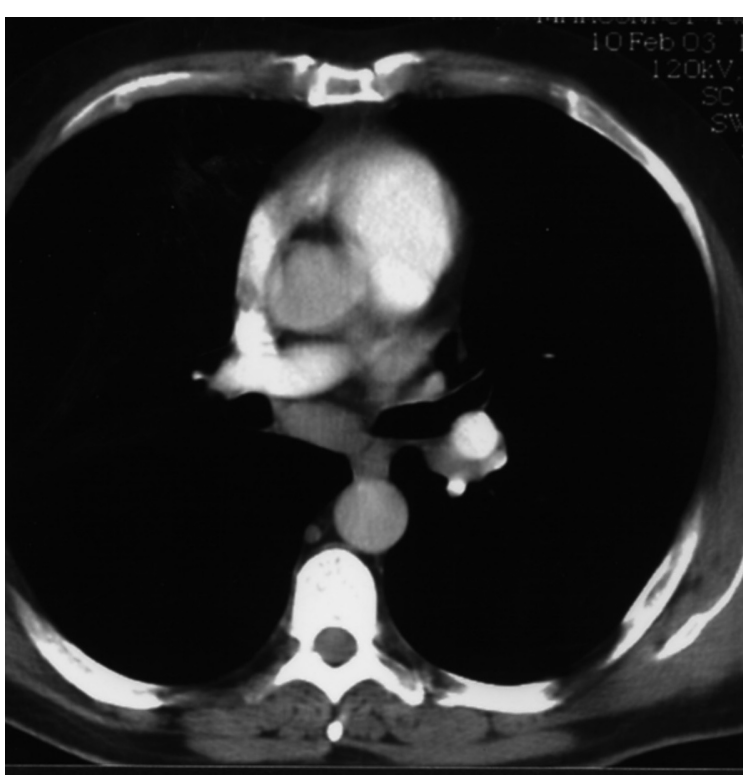

Figure 7 : Examen tomodensitométrique pulmonaire. Axial view CT Scan of lungs

thies, très fréquentes, ont le plus souvent une localisation médiastinale ou hilaire, plus rarement superficielles et diffuses, en particulier dans la région cervicale. Des lésions extra-pulmonaires s'observent dans 40 à $50 \%$ des cas [1] : les lésions cutanées se traduisent habituellement par des « sarcoïdes » nodulaires avec des « lupoïdes » périphériques jaunâtres; les manifestations buccales [2,3] sont constituées de nodules, de papules [4] ou d'ulcérations [5] indolores, rouge sombre, avec présence de «lupoïdes ». Ces manifestations buccales sont le plus souvent latentes et elles peuvent intéresser les lèvres [6], les joues, la langue [7,18], le plancher buccal, le palais [3], le voile du palais, le pharynx [8] ou l'os alvéolaire [6]. Dans $50 \%$ des cas de sarcoïdose, il y a une atteinte des glandes salivaires accessoires, plus rarement des glandes salivaires principales, réalisant parfois une parotidite bilatérale « froide » se traduisant par une parotidomégalie (observée dans 1-4 \% des cas) avec éventuellement une hyposialie. La sarcoïdose peut aussi comporter à une atteinte rénale (qui amène souvent à la découverte de l'affection), hépatique, gastro-intestinale [9], articulaire, nerveuse avec éventuellement des épisodes de paralysie faciale [11], ophtalmique [10] et lacrymale. Des affections systémiques sont parfois associées : syndrome de 
Löfgren, érythème noueux ou lupus pernio. Les granulomes évoluent soit vers la résorption, soit vers la transformation fibreuse irréversible. La maladie, qui peut régresser spontanément, répond habituellement favorablement à la corticothérapie. Bien que considérée comme bénigne, près de $5 \%$ des patients vont décéder principalement de complications cardio-respiratoires (hémoptysie sur aspergillome...) ou neurologiques. La découverte de la sarcoïdose est rarement le fait de l'odonto-stomatologiste [12]. En effet, la rareté des atteintes buccales concourt à une méconnaissance de l'affection et les lésions manquent de spécificité.

La symptomatologie et l'imagerie du cas présenté évoquent une cellulite chronique d'origine dentaire. Néanmoins, la durée d'évolution, l'absence d'adénopathie cervicale, de modification du revêtement cutané, de comblement vestibulaire et d'hyperthermie constituent autant d'éléments discordants. Dans ce contexte, le traitement consistant à supprimer les foyers infectieux locaux reste cohérent. Mais I'évolution atypique, avec persistance de la tuméfaction, impose des investigations complémentaires. L'examen anatomopathologique du prélèvement jugal évoque le diagnostic de sarcoïdose, tandis que celui des glandes salivaires accessoires élimine notamment une amylose, une maladie de Crohn, un syndrome de Melkersson-Rosenthal ou une maladie de Gougerot-Sjögren.

Le diagnostic de sarcoïdose repose habituellement sur la biopsie [13]. Le diagnostic définitif de sarcoïdose impose une démarche s'appuyant sur la conjonction de divers éléments d'orientation et d'exclusion. La découverte d'anomalies histologiques avec des lésions granulomateuses tuberculoïdes, sans nécrose caséeuse, est très évocatrice de la maladie. Cet élément doit être confirmée par les données de la clinique, de l'imagerie ou de la biologie (Kveim [14], anergie, calcémie [15], enzyme de conversion, VS, acide urique, phosphatases alcalines, anémie discrète, augmentation globale des immunoglobulines). ॥ convient aussi d'exclure les autres affections granulomateuses.

Dans le cas présenté, le tableau clinique comporte une tuméfaction jugale accompagnée d'un trismus. Ce dernier peut être rapproché des contractures observées par Walter [16] sur les muscles longs. Les localisations musculaires de la sarcoïdose semblent peu nombreuses, une revue de la littérature décrit 79 cas de sarcoïdomyopathie, constituant dans $44 \%$ des cas une forme isolée [17]. Des localisations buccales sont décrites par Serrat [18], linguales par Nagata [7], Blinder décrit des cas de sarcoïdose comportant une gingivite érythémateuse douloureuse, avec des ulcérations superficielles dans la région antérieure, des localisations labiales et des indurations linguales sous-muqueuses [6]. Long rapporte aussi des cas d'atteintes sinusiennes [19].

Le cas rapporté présente des adénopathies médiastinales, des bronchectasies et des micronodules pulmonaires, ce qui est relativement classique. En revanche, I'absence d'autres éléments d'orientation, comme un taux élevé de l'enzyme de conversion ou du calcium plasmatique, confirme le grand polymorphisme de cette affection. Enfin, l'absence de BK dans les crachats élimine une tuberculose.

La suspicion d'une sarcoïdose doit faire envisager le diagnostic différentiel différemment selon le tableau clinique et radiologique, et la présence de lésions granulomateuses [5, 20]. II faut évoquer : - devant la présence de granulomes : une tuberculose, plus rarement une histoplasmose, une bérylliose (exposition professionnelle ou traitement par interféron), une aspergillose (sur les formes pulmonaires fibrosées) ou une cryptococcose ;

- devant des granulomes avec adénopathies médiastinales : rarement une tuberculose où les adénopathies sont plutôt unilatérales, ou un lymphome surtout si les adénopathies sont antérieures ;

- devant des granulomes avec atteinte pulmonaire : une pneumopathie d'hypersensibilité ou une pneumopathie médicamenteuse ;

- devant des granulomes et une atteinte extrathoracique : la lèpre, la syphilis, la lymphogranulomatose d'inoculation, une granulomatose médicamenteuse, une maladie de Crohn, un syndrome de Melkersson-Rosenthal, une chéilite granulomateuse de Miescher, un cancer broncho-pulmonaire, une maladie de Whipple...; médecine

buccale

chirurgie

buccale

VOL. $10, \mathrm{~N}^{\circ} 2$ 2004

page 87 
médecine

buccale

chirurgie

buccale

VOL. $10, \mathrm{~N}^{\circ} 2$ 2004

page 88
- devant des adénopahies médiastinales sans granulomes : surtout des métastases de cancers solides au stade clinique ;

- devant une atteinte pulmonaire sans granulome: une lymphangite carcinomateuse surtout secondaire à un cancer médullaire de la thyroïde, une histiocytose $X$, une fibrose interstitielle diffuse primitive, une amyloïdose pulmonaire diffuse.

En ce qui concerne le traitement, le patient a réagi favorablement à une corticothérapie générale avec disparition de la tuméfaction jugale en un mois. Actuellement, on considère que l'utilisation des corticoïdes est seulement justifiée pour contrôler les manifestations cliniques invalidantes et/ou mettant en jeu le pronostic fonctionnel. La corticothérapie est donc indiquée lorsqu'il existe une symptomatologie générale, une atteinte bronchopulmonaire sévère, des localisations extrarespiratoires menaçantes, en particulier neurologique, cardiaque, ophtalmologique ou rénale (associée à une hypercalcémie). Par ailleurs, ce traitement est en partie palliatif car il ne prévient ni la fibrose pulmonaire ni les altérations définitives de la fonction pulmonaire [21] ; il aurait même un effet délétère à long terme. II existe un consensus pour une dose d'attaque de $0,5 \mathrm{mg} \cdot \mathrm{kg}^{-1} \cdot \mathrm{j}^{-1}$ de prednisone ou de prednisolone pendant 3 mois, avec parfois $1 \mathrm{mg}^{\mathrm{kg}} \mathrm{kg}^{-1} . \mathrm{j}^{-1}$ le premier mois. Lorsque le résultat est favorable, on prescrit un traitement d'entretien pour une durée minimale de 15 mois avec une dose lentement dégressive par plateaux de 3 mois. En cas de reprise évolutive, il suffit généralement de reprendre la posologie antérieure pour contrôler la maladie. Les corticoïdes locaux - collyres, pommades, crèmes - peuvent être utiles pour le traitement des atteintes superficielles. Les aérosols ont une efficacité discutée.

Des thérapeutiques alternatives doivent être envisagées devant une sarcoïdose grave, rebelle à la corticothérapie ou lorsque la corticothérapie est responsable d'effets secondaires sévères. Le méthotrexate peut être efficace mais provoque des effets secondaires pulmonaires et hépatiques. A faibles doses, il agirait plus par son effet antiinflammatoire que son action anti-métabolique : il est indiqué dans les sarcoïdoses cutanées sévères comme le lupus pernio. II semble efficace dans les atteintes oculaires et il est utilisé pour les localisations pulmonaires et rénales. Le traitement, à la posologie de $10 \mathrm{mg} \cdot \mathrm{sem}^{-1}$ en traitement d'attaque, puis de $5 \mathrm{mg} \cdot \mathrm{sem}^{-1}$, dure en moyenne 2 ans. Son arrêt est souvent suivi de rechutes, d'où la nécessité d'un traitement prolongé. Les antipaludéens de synthèse sont efficaces pour les localisations cutanées disgracieuses. Walter a essayé avec succès le thalidomide à la dose de 50 mg..$^{-1}[16]$; il suggère la possibilité de l'utiliser pour les sarcoïdoses rebelles mais son efficacité reste l'objet de controverses. Meyerle propose d'utiliser l'infliximab, un anticorps monoclonal inhibant sélectivement le TNF-alpha [22]. D'autres traitements ont étés essayés : la ciclosporine, le chlorambucil, le cyclophosphamide, I'azathioprine, I'isotrétinoïne, l'allopurinol, le Bactrim ${ }^{\circledR}$, la puvathérapie, le laser et la mélatonine, mais il s'agit d'études non contrôlées, et leur efficacité reste à démontrer.

Dans le cas présenté, on observe au cours du traitement l'apparition d'une asthénie, d'une irritabilité, d'un syndrome anxio-dépressif, d'une polyurie et d'une polydipsie. II s'agit d'effets secondaires bien connus de la corticothérapie. Après un effet euphorisant initial, la corticothérapie peut entraîner un syndrome dépressif. Le diabète corticoinduit est un phénomène fréquent, surtout lorsqu'il existe une prédisposition familiale. Ces suites confirment les réserves évoquées précédemment concernant le traitement, pourtant dans ce cas il ne semble pas exister d'alternative sinon une recherche de la dose minimale efficace. Enfin, un retard thérapeutique peut aboutir à des situations médicales irréversibles, ainsi l'odonto-stomatologiste doit assurer un dépistage précoce dans le cas de manifestations bucco-faciales. 


\section{RÉFÉRENCES}

1 - Belfer MH., Stevens W. Sarcoidosis: A primary care review. Am Fam Physician 1998; 68 : 2041-51.

2 - HILLERUP S. Diagnosis of sarcoidosis from oral manifestation. Int J Oral Surg $1976 ; 5:$ 95-9.

3 - COMPADRETTI GC, NANNINI R, TASCA I. Isolated tonsillar sarcoidosis manifested as asymmetric palantine tonsils. Am J Otolaryngol 2003 ; 24 : 187-90.

4 - Hildebrand J, Plezia RA, Rao SB. Sarcoidosis. Report of two cases with oral involvement. Oral Surg Oral Med Oral Pathol 1990 ; $69: 217-22$.

5 - Piattelli A, Favia GF, Di Alberti L. Oral ulceration as a presenting sign of unknown sarcoidosis mimicking a tumour: report of 2 cases. Oral Oncol 1998 ; 34 : 427-30.

6 - Blinder MD, YAhatom R, TAICHER S, HAShomer T. Oral manifestations of sarcoidosis. Oral Surg Oral Med Oral Pathol Oral Radiol Endod 1997 ; 83 : 459-61.

7 - Nagata Y, Kanekura T, Kawabata H, Shimomal K, HIGaSH Y, SetOYAMA M, KanZAKI T. A case of sarcoidosis involving the tongue. J Dermatol $1999 ; 26: 666-70$.

8 - ONISHI Y, IMAI Y, TOJIMA H, NAKAJIMA K, TAKAHASHI A. Systemic sarcoidosis with significant granulomatous swelling of the pharyngeal tonsil. Intern Med 1998 ; 37 : $157-60$

9 - Fireman Z, Sterniberg A, Yarchovsky Y, Abu-Much S, Coscas D, Topilsky M, Fireman E, Groisman GM. Multiple antral ulcers in gastric sarcoid. J Clin Gastroenterol $1997 ; 24: 97-9$.

10 - IMES RK, REIFSCHNEIDER JS, O'CONNOR LE. Systemic sarcoidosis presenting initially with bilateral orbital and upper lid masses. Ann Ophthalmol $1988 ; 20$ : 466-9.

11 - Hanazawa T, Terada, Konno A. Sarcoidosis of the head and neck. Nippon Rinsho 2002 ; 60 : 1813-7.
12 - Gold RS, SAGER E. Oral sarcoidosis: review of the literature. J Oral Surg $1976 ; 34: 237-44$.

13 - KISSI L, BENYAHYA I. Sarcoïdose : à propos d'un cas. Actual odontostomatol $2003 ; 222: 131-7$.

14 - Greer RO JR, SAnger RG. Primary intraoral sarcoidosis. J Oral Surg 1977 ; 35 : 507-9.

15 - Dorfler H, Conca W, Remberger K. Granulomatous gastritis of the antrum in generalized sarcoidosis. Klin Wochenschr $1986 ; 64: 1139-43$.

16 - Walter MC, Lochmuller H, Schlotter-Weigel B, MEINDL T, MULLER-FELBER W. Successful treatment of muscle sarcoidosis with thalidomide. Acta Myol 2003; $22: 22-5$.

17 - DE Pree C, Dessibourg CA. Sarcoidosis: apropos of a case of isolated myopathy. Schweiz Rundsch Med Prax $1989 ; 78: 557-9$.

18 - Serrat Soto A, Lobo Valentin P, Redondo Gonzalez lm, Sanz Santa Cruz C, Verrier Hernandez A. Oral sarcoidosis with tongue involvement. Oral Surg Oral Med Oral Pathol Oral Radiol Endod 1997 ; 83 : 668-71.

19 - LONG CM, SMitH T, LOEHRL TA, KomorowsKI RA, TOOHILL RJ. Sinonasal disease in patients with sarcoidosis. Am J Rhinol $2001 ; 15: 211-5$.

20 - DeLUKE DM, ScIUBBA JJ. Oral manifestations of sarcoidosis: report of a case masquerading as a neoplasm. Oral Surg Oral Med Oral Pathol 1985 ; 59 : 184-8.

21 - SouISsI R. Site internet du service de dermatologie de I'hôpital Habib Thameur. Adresse : http://www.stmi.org.tn/Index.htm . Date de visite : 16/12/2003.

22 - MeYerLE JH, SHORR A. The use of infliximab in cutaneous sarcoidosis. J Drug Dermatol 2003 ; 2 : 413-4.

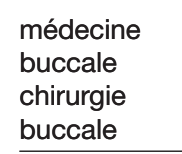

VOL. $10, \mathrm{~N}^{\circ} 2$ 2004

page 89 